\title{
Efficient transfer of PSA and PSMA cDNAs into DCs generates antibody and $T$ cell antitumor responses in vivo
}

\author{
Jeffrey A Medin, ${ }^{1,2}$ Sheng-Ben Liang, ${ }_{3}^{1}$ Jeannie Whit-Shan Hou, ${ }^{3}$ Leslie S Kelley, ${ }^{4}$ \\ David J Peace, ${ }^{4}$ and Daniel H Fowler ${ }^{3}$
}

\author{
${ }^{1}$ Division of Experimental Therapeutics, Ontario Cancer Institute, Toronto, Ontario, Canada M5G 2MI; \\ ${ }^{2}$ Department of Medical Biophysics, University of Toronto, Toronto, Ontario, Canada, M5G 2M1; \\ ${ }^{3}$ Experimental Transplantation and Immunology Branch, National Cancer Institute, Bethesda, Maryland \\ 20892, USA; and ${ }^{4}$ Section of Hematology/Oncology, Department of Medicine, University of Illinois at \\ Chicago, Chicago, Illinois 60607, USA.
}

\begin{abstract}
Gene therapy for prostate cancer may be realized through transduction of whole genes, such as PSA or PSMA, into immunotherapeutic dendritic cells (DCs). An oncoretroviral vector encoding human PSMA and a bicistronic oncoretroviral vector encoding human PSA and cell surface CD25 cDNAs were constructed. Remarkably, transfer of PSA/CD25 or PSMA cDNA during murine hematopoietic cell differentiation into DCs occurred with approximately $80 \%$ efficiency. In vitro, transduced DCs retained allostimulatory function and primed syngeneic $\mathrm{T}$ cells for tumor antigen-specific IFN- $\gamma$ secretion. In test experiments designed to elucidate mechanisms in vivo, syngeneic recipients of transduced DCs had increased anti-human PSA antibody titers and tumorspecific $\mathrm{CD}^{+}{ }^{+} \mathrm{T}$ cell IFN- $\gamma$ secretion with no detectable immune response to CD25. Gene-modified DC recipients had increased protection from specific tumor challenge for at least 18 weeks post-vaccination. DC vaccination also protected both male and female recipients. Gene-modified DC vaccination mediated regression of established, specific gene-expressing, TRAMP-C1 prostate cancer cell tumors. These findings indicate that antibody and cellular responses generated through PSA and PSMA gene transfer into DC yielded protective immunity, thereby providing further preclinical support for the implementation of immuno-gene therapy approaches for prostate cancer.
\end{abstract}

Cancer Gene Therapy (2005) 12, 540-551. doi:10.1038/sj.cgt.7700810

Published online 28 January 2005

Keywords: dendritic cells; immunotherapy; onco-retrovirus

Tmmunotherapy may represent a valid approach for 1 prostate cancer therapy. ${ }^{1-3}$ To this extent, we have initiated an evaluation of clinically relevant retrovirusmediated gene transfer of prostate cancer-associated antigens into potentially therapeutic dendritic cells (DCs). DCs are derived from hematopoietic cells of the bone marrow (BM) and stimulate both $\mathrm{B}$ and $\mathrm{T}$ lymphocytes. ${ }^{4}$ DCs present processed peptides in the context of MHC Class I and Class II molecules to cytotoxic $\mathrm{T}$ lymphocytes (CTLs) and helper $\mathrm{T}$ cells, respectively, and express high levels of adhesion signals and costimulatory molecules. ${ }^{5}$ Importantly, DC generation and maturation are inhibited by prostate cancer cells ${ }^{6}$ and DCs are eliminated by apoptosis ${ }^{7}$ in the prostate tumor mass. ${ }^{8}$ As such, suboptimal DC function may represent an important mechanism for the formation of self-tolerance to this cancer. Thus, it is possible that

Received July 14, 2004

Address correspondence and reprint requests to: Dr Jeffrey $A$ Medin, PhD, University Health Network, 67 College Street Room 406, Toronto, Ontario, Canada M5G $2 \mathrm{MI}$.

E-mail:jmedin@uhnres.utoronto.ca transplantation of functional DCs may help to break this tolerance, especially if these cells are engineered to overexpress and present peptides from specific prostate tumor antigens.

Retroviruses as a class offer the possibility of sustained expression of the transferred gene in parent and progeny cells along with low reactivity or toxicity to the delivery agent. Since we and others have shown that hematopoietic stem/progenitor cells derived from the BM can be transduced with recombinant oncoretroviral vectors, 9,10 we sought to transduce these cells with vectors that engineer expression of human prostate-specific antigens (PSAs) and differentiate them into functional DCs. Although no prior studies appear to exist relating to retroviral transfer of prostate-associated tumor antigens into this cell population, other investigators have utilized clinically relevant oncoretroviruses to transfer other genes into functionally defined DCs. ${ }^{1-14}$ Lastly, it has also been shown recently that DC cell expansion can even occur under appropriate conditions in culture ${ }^{15}$ possibly providing more immune effector cells for transplant.

In this study, we have focused on two primary identified human prostate antigens, PSA and prostate- 
specific membrane antigen (PSMA). PSA is a member of a subfamily (glandular kallikreins) of serine proteases. It is secreted by the ductile epithelium of the prostate and exists in free and complexed forms. PSA functions to liquefy seminal fluid and has been widely used as a clinical marker for the diagnosis and staging of prostate cancer. ${ }^{16}$ It has been shown that human cytotoxic $\mathrm{T}$ lymphocytes (CTLs) respond to peptides derived from PSA $^{17}$ and that intramuscular immunization of mice with plasmid DNA encoding PSA also leads to potent CTL responses to specific tumor cells. ${ }^{18}$ Furthermore, a preponderance of antibody responses were found to be directed to PSA antigens when general mRNA from a prostate tumor line was used to transfect autologous DCs. ${ }^{19}$ In sum, these studies indicate that PSA is a valid antigen for potential prostate cancer immunotherapy strategies.

PSMA is a glycoprotein that exists both in a transmembrane and a secreted form. ${ }^{20}$ The function of PSMA is not well defined, although the protein demonstrates folate hydrolase activity and nucleotide homology to a neurocarboxypeptidase and to transferrin receptor. ${ }^{21}$ In addition to their different biological roles and biochemical characteristics, PSA and PSMA are often reciprocal in their expression in prostate tumors. For example, higher levels of PSMA expression is associated with progression of androgen-insensitive prostate cancer, while levels of PSA were not found to be correlative. ${ }^{22}$ While the expression of both PSA and PSMA is fairly tissue specific, PSMA has also been detected in endothelial cells of a wide variety of tumor neo-vasculature. ${ }^{23}$ PSMA has been used in clinical gene transfer-based immunotherapy strategies as Mincheff et $\mathrm{al}^{24}$ have recently tested plasmid and adenoviral constructs that engineer expression of PSMA in Phase I/II trials.

In our experiments, we demonstrate that effective recombinant oncoretroviral gene transfer vectors can be generated that engineer overexpression of human PSA and PSMA in efficient murine DCs. We found that the whole prostate gene-modified DC generated both antibody and $\mathrm{CD} 8{ }^{+} \mathrm{T}$ cell host immune responses, therefore suggesting that this DC approach may provide tumorassociated antigen presentation along both Class II and Class I MHC presentation pathways. These data, when combined with the observed protection from tumor challenge and reduction of pre-existing tumors, indicate that this clinically relevant oncoretroviral-transduced DC immunotherapy approach represents a promising area of research into the development of gene therapy strategies for prostate cancer.

\section{Materials and methods}

Prostate antigen vectors and packaging cell lines

The pUMFG/PSMA vector was engineered as follows: Plasmid pGEX4T.2/PSMA was obtained from Dr Charles Young (Mayo Clinic). A novel NcoI restriction enzyme site was introduced near the start of the coding sequence by primer-directed mutagenesis using the Quik-
Change $^{\mathrm{TM}}$ kit (Stratagene, La Jolla, CA). The coding sequence for human PSMA was then subcloned into the retroviral transfer vector plasmid pUMFG (Takenaka et al, 1999) to give pUMFG/PSMA, which was fully confirmed by restriction enzyme digests and by DNA sequencing. For vector $\mathrm{pUMFG/PSA/IRES/huCD25,} \mathrm{the}$ human PSA cDNA was amplified from an mRNA library derived from LNCaP cells (kindly supplied by Dr Mark Heller, UIC). The sequence was subcloned into a shuttle vector containing the sequences for the internal ribosome entry site (IRES) from the encephalomyocarditis virus and the human interleukin-2 receptor $\alpha$ chain (huCD25). The resulting PSA/IRES/huCD25 bicistronic construct was then subcloned into pUMFG. This gives plasmid pUMFG/PSA/IRES/huCD25. The fidelity of this vector was also checked as above. Two nucleotides of the human PSA-coding sequence were found to differ from the published sequence ${ }^{25}$ and represent mutations (97ProThr, 136Met-Val) or polymorphisms. These nucleotides were changed to the published sequence ${ }^{25}$ by QuikChange $^{\mathrm{TM}}$ mutagenesis (Stratagene) using appropriate DNA primers and resequenced in both orientations for confirmation.

The plasmid vector constructs were transfected using calcium phosphate ${ }^{26}$ in separate reactions into ecotropic and amphotropic oncoretroviral vector packaging cell lines E86 and PG13, respectively. Packaging cell lines were grown as described. ${ }^{10,26}$ Transfected cell pools were selected in G418 (Life Technologies, Gaithersburg, MD) for neomycin phosphotransferase expression from a cotransfected plasmid (pGT-N28; New England Biolabs Inc., Beverley, MA). PSMA and huCD25 cell surface expression were measured directly on pooled cell lines by flow cytometry (see below). Single-cell clones were then isolated from each pool of producer cells by flow sorting (top $0.5 \%-2.5 \%$ positive cells) and subsequent limiting dilution cultures. Clones were then titered using serial dilutions of filtered supernatant onto NIH3T3 or HeLa cells. PSMA and huCD25 cell surface expression on recipient cells were again measured by flow cytometry. To discount bias, clarified overnight supernatants from transfected and selected PSA packaging cell line clones were tested blindly as serum samples for secreted human PSA levels at the UIC Hospital. Clones selected for use in this study were named E86/PSA/IRES/huCD25 \#21/1520, PG13/PSA/IRES/CD25\#21/15-17, E86/PSMA\#65, and PG13/PSMA\#25. Titers of these producer clones were estimated to be $\sim 10^{6}$ productive infectious units $/ \mathrm{ml}$ for the E86 clones and $\sim 10^{5}$ productive infectious units/ $\mathrm{ml}$ for the PG13 clones. A direct assay ${ }^{26}$ was used to determine that no replication competent retrovirus (RCR) was present. For Expt. 3 (see below), the $\alpha$-gal A/huCD25 ecotropic packaging cell line from Qin et $\mathrm{al}^{10}$ was used.

\section{Transduction and maturation of murine BM cells}

Mouse BM cells were collected from the femurs and tibiae of female and male BALB/c donors. Cells were pelleted by centrifugation and BMMC were isolated using Nyco$\operatorname{Prep}^{\text {TM }}$ A 1.077 (AXIS-SHIELD PoC AS, Oslo, Norway), 
and incubated at $37^{\circ} \mathrm{C}$ with $5 \% \quad \mathrm{CO}_{2}$ for 2 hours to remove adherent cells. These cells were then transduced five times with filtered supernatants from ecotropic producer clones in the presence of $20 \mathrm{ng} / \mathrm{ml}$ of recombinant murine (rm) IL-4 and rmGM-CSF (R\&D Systems Inc., Minneapolis, MN) over a 5-day period. In an attempt to facilitate DC maturation, ${ }^{4}$ the cells were then incubated with $10 \mathrm{ng} / \mathrm{ml} \mathrm{mTNF}-\alpha$ (R\&D Systems, Inc.) for 1 day with or without rmIL-4 and rmGM-CSF.

\section{Antibodies and flow cytometric analyses}

The mouse anti-human PSMA mAb PEQ226.5. was kindly provided by Hybritech Inc. (San Diego, CA). Fluorescein isothiocyanate (FITC)-conjugated rat antimouse CD3, CD11b, CD25, CD40, CD86 (B7-2) and CD244 (NK), FITC-conjugated hamster anti-mouse CD11c and CD54 (ICAM-1), FITC-conjugated mouse anti-mouse I-A ${ }^{\mathrm{d}}$ MHC Class II alloantigen, R-phycoerythrin (PE)-conjugated mouse anti-human CD25 (IL$2 \mathrm{R} \alpha$ ), PE-conjugated rat anti-mouse CD19, IFN- $\gamma$, GR-1, and purified rat anti-mouse CD16/CD32 and CD80 (B71) were purchased from BD Biosciences Pharmingen (San Diego, CA). FITC- and PE-conjugated goat-F $\left(\mathrm{ab}^{\prime}\right)_{2}$ antimouse $\mathrm{IgG}_{1}$ were purchased from Southern Biotechnology Associates, Inc. (Birmingham, AL). Purified mouse anti-human CD25 was purchased from BD Biosciences Immunocytometry Systems (San Jose, CA). Rat antimouse CD205 (DEC205) was purchased from Serotec Ltd. (Kidlington, Oxford, UK). Matched isotype control antibodies were purchased from BD Biosciences Pharmingen, except for mouse $\operatorname{IgG}_{1} \kappa$ (MOPC-31C; SigmaAldrich Inc., St Louis, MO) and purified rat $\operatorname{IgG} 2 \mathrm{a}$ (Serotec).

For staining, cells were collected and washed in D-PBS (BioWhittaker Inc., Walkersville, MD). Indiscriminate antibody binding was blocked with $20 \%$ normal rabbit serum (Sigma) in D-PBS (Blocking Solution) for 20 minutes at $4{ }^{\circ} \mathrm{C}$, at a dilution of $50 \mu \mathrm{l} / 10^{5}-10^{6}$ cells. Primary antibody was then added after washing in $\mathrm{D}$ PBS and incubated with the cells for 30 minutes at $4{ }^{\circ} \mathrm{C}$. The cells were washed with Staining Buffer $(5 \mathrm{mM}$ EDTA/1\% BSA (Sigma) in PBS) and collected by centrifugation at $500 \mathrm{~g}$ for 5 minutes at $4{ }^{\circ} \mathrm{C}$. The cells were resuspended in $50 \mu \mathrm{l}$ of Blocking Solution with appropriate secondary antibodies and incubated for 15 minutes at $4^{\circ} \mathrm{C}$, washed twice with Staining Buffer, collected by centrifugation at $500 \mathrm{~g}$ for 5 minutes, and resuspended into $0.2-1 \mathrm{ml}$ of Staining Buffer for flow cytometric analysis. Propidium iodide (Sigma) at a $1 \mu \mathrm{g} /$ $\mathrm{ml}$ final concentration, or 7-aminoactinomycin D (Sigma) at a $1 \mu \mathrm{g} / \mathrm{ml}$ final concentration, was added to exclude dead cells. Flow cytometric analyses were performed using a FACSCalibur $^{\mathrm{TM}}$ (BD Biosciences). Isotype controls were used to set gatings in experiments and for subtraction of background staining levels.

\section{Allogeneic $M L R$}

BM-derived cells (transduced and nontransduced) or freshly prepared splenocytes were isolated from BALB/c donors. Splenocytes were extruded from intact spleens with dissecting needles and suspended in D-PBS. The cells were passed through a fine mesh screen, washed, and suspended in culture medium (see below). The DCs and splenocytes were then irradiated at 2000 rads, and plated in serial dilutions along with $2 \times 10^{5}$ nonstimulated allogeneic splenocytic $\mathrm{T}$ cells (isolated from $\mathrm{C} 57 \mathrm{BL} / 6$ donors; RBC-depleted using lysis buffer (BioRad Aquapure, Hercules, CA) and B-cell-depleted by goat antimouse IgG negative selection (Dynal Inc., Lake Success, NY)) in $200 \mu \mathrm{l}$ of growth medium: RPMI-1640 culture media supplemented with $10 \%$ heat-inactivated fetal calf serum $(\mathrm{FCS})$, pen/strep/glutamine, $1 \times$ nonessential amino acids, $1 \mathrm{mM}$ sodium pyruvate (BioFluids), plus $1.25 \mu \mathrm{g} / \mathrm{ml}$ amphotericin $\mathrm{B}$ and $50 \mu \mathrm{g} / \mathrm{ml}$ gentamicin sulfate (Life Tech) in 96-well tissue culture plates (flatbottomed) for 3.5 days. The cultures (in triplicate for each data point) were incubated at $37^{\circ} \mathrm{C}$ with $5 \% \quad \mathrm{CO}_{2}$. Cultures were then pulsed with $1.0 \mu \mathrm{Ci} /$ well $\left[{ }^{3} \mathrm{H}\right]$ thymidine (Amersham Pharmacia Biotech, Piscataway, NJ) for 6 hours. Following that, $\left[{ }^{3} \mathrm{H}\right]$ thymidine incorporation as a measure of proliferation was determined using a TopCount $\beta$ scintillation counter (Packard Instrument Corp., Meriden, CT).

\section{IL-2-mediated proliferation assay for tranduced and control mouse cells}

Mouse T cells and DC-like cells were isolated, cultured, and harvested as described above. Cells were enriched for murine CD11c expression using N418 Microbeads (Miltenyi Biotec, Auburn, CA) or for CD90 expression using Thy1.2 Microbeads (Miltenyi), according to the manufacturer's instructions. In all, $1 \times 10^{5}$ CD11c-enriched cells or $2 \times 10^{6} \mathrm{~T}$ cells per $\mathrm{ml}$ were seeded into $100 \mu \mathrm{l}$ of growth medium (as above) with $0,10^{3}, 10^{4}$, or $10^{5} \mathrm{IU} / \mathrm{ml}$ of mouse IL-2 (R\&D Systems) and incubated for 2 days at $37^{\circ} \mathrm{C}$ with $5 \% \mathrm{CO}_{2}$ in 96 -well plates. Next, $10 \mu \mathrm{l} /$ well MTT labeling solution from the MTT Cell Proliferation Kit I (Roche) was added and incubated for 4 hours at $37^{\circ} \mathrm{C}$ with $5 \% \mathrm{CO}_{2}$. Solubilization solution $(100 \mu \mathrm{l} /$ well $)$ from the kit was then added and incubated at $37^{\circ} \mathrm{C}$ with $5 \% \mathrm{CO}_{2}$. The 96-well plate was then read at $570 \mathrm{~nm}$ using an ELISA Plate Reader with a reference wavelength of $650 \mathrm{~nm}$.

\section{Creation of human prostate antigen-specific murine tumor cell lines}

MOPC-315 murine (strain: BALB/c) plasmacytoma cells $^{27}$ were supplied by Dr Margalit Mokyr at UIC. MOPC cells were grown in DMEM with $10 \%$ FCS, pen/ strep, glutamine, HEPES Buffer Solution, Nonessential Amino Acids Solution (all GibcoBRL, Gaithersburg, MD), and $0.7 \mathrm{mM} \beta$-mercaptoethanol (Sigma). MOPC cells were infected with E86-derived supernatants of human PSA, PSMA, and $\alpha$-gal A, yielding MOPC/PSA/ huCD25, MOPC/PSMA, and MOPC/ $\alpha$-gal A/huCD25 cell lines, respectively. Infected MOPC lines were enriched by repeated immunoaffinity chromatography using pure mouse anti-huCD25 or anti-PSMA antibodies, goat 
anti-mouse IgG microbeads (Miltenyi), and MS + RS + columns (Miltenyi) according to the manufacturer's protocol. A high percentage (up to $90 \%$ ) of MOPC/ PSA/huCD25 and MOPC/ $\alpha$-gal A/huCD25 cells stained positive for huCD25 expression. Likewise, a high percentage (up to $95 \%$ ) of MOPC/PSMA cells stained positive for PSMA expression.

TRAMP-C1 is a murine SV40 large $\mathrm{T}$ antigenexpressing transgenic prostate cancer cell line of $\mathrm{Bl} / 6$ origin. $^{28}$ TRAMP-C1 cells were grown in DMEM high glucose with L-glutamine and without sodium pyruvate media (SIGMA) supplemented with 5\% Nu-serum IV (BD Biosciences), 5\% FCS, $5 \mu \mathrm{g} / \mathrm{ml}$ human insulin (SIGMA), $25 \mathrm{U} / \mathrm{ml}$ penicillin-streptomysin (CAMBREX), and $10^{-8} \mathrm{M}$ dihydrotestosterone (SIGMA).

For creation of human PSA-expressing murine prostate tumor cell lines, TRAMP-C1 cells were infected with E86derived PSA/IRES/huCD25 vector supernantant and enhanced GFP (enGFP) supernatant as a control. PSAinfected TRAMP-C1 (TRAMP-C1/PSA/IRES/huCD25) cells were enriched in our laboratory by repeated magnetic cell immunosorting using pure mouse antihuCD25 (BD), goat anti-mouse IgG microbeads (Miltenyi), and MS + RS + columns (Miltenyi) according to the manufacturer's protocol. EnGFP-infected TRAMPC1 (TRAMP-C1/enGFP) cells were enriched by flow cytometric sorting. High percentages of TRAMP-C1/ PSA/IRES/huCD25-positive cells $(95 \%)$ and TRAMPC1/enGFP (98\%) were obtained and used for the experiment.

\section{Primary in vitro $T$ cell sensitization assay}

PSA- or PSMA-transduced BALB/c cells were prepared as above. Syngeneic Balb/c splenocytes were isolated and further enriched for $\mathrm{T}$ cells by $\mathrm{B}$ cell depletion using goatanti-mouse IgG bioparticles (Polysciences Inc., Warrington, PA). A co-culture was then performed at a T cell: DC ratio of 10:1. Culture media consisted of RPMI-1640 supplemented with 10\% FCS (Gemini BioProducts; Woodland, CA), recombinant human (rh) IL-2 (1000 IU/ml; NCI BRB Preclinical Repository, Rockville, MD), rhIL-7 (20 ng/ml; Peprotech, Rocky Hill, NJ), rm IL-4 (1000 IU $/ \mathrm{ml} ;$ Peprotech), and $3.3 \mu \mathrm{M} N$-acetyl cysteine (Geneva Pharmaceuticals, Dayton, NJ). To augment $\mathrm{T}$ cell number and enhance effector function, $\mathrm{T}$ cells were harvested from culture at day 4 and restimulated in a polyclonal manner with tosylated magnetic beads (Dynal) coated with anti-CD3 (clone 145-2C11; PharMingen) and anti-CD28 antibodies (clone 37.51; PharMingen) at a $\mathrm{T}$ cell to bead ratio of $3: 1$. The method for preparation of antibody-coated beads has been previously detailed. ${ }^{29}$ At day 10 of culture, the expanded T cells were harvested, brought to $1 \times 10^{6}$ cells $/ \mathrm{ml}$, placed into a 24-well plate, and stimulated with either unmodified MOPC tumor cells, PSA/huCD25-, or PSMAtransduced MOPC cells (each at a 10:1 T cell to tumor cell ratio), or repeat anti-CD3/anti-CD28 bead stimulation (at a 3:1 T cell to bead ratio). The 24 hour supernatants were harvested, and tested for cytokine content by commercially available two-site ELISA kits (IFN- $\gamma$, IL-4, and IL-10 kits were from BioSource, Camarillo, CA; IL-2 and IL-5 kits were from R \& D Systems).

\section{Murine transplantation studies /tumor challenge/tumor reduction}

Female and male BALB/c mice, 7- to 10-week-old, were purchased from Charles River Laboratories (Montreal, Canada). The mice were housed in a specific pathogenfree environment in the animal facility at the UHN or UIC and studies were performed under Animal Care Committee approval. Nonconditioned recipient mice were immunized by i.p. delivery of $4 \times 10^{5}$ cells per mouse of DC/NT, DC/PSA/huCD25, or DC/PSMA, separately. After 4 weeks, a second immunization was performed similarly. At that point four mice were randomly selected from each group and spleen-derived $T$ cells were obtained for functional analyses (see below). After 1 week (Expts. $1 \& 3$ ) or 18 weeks (Expt. 2), tumor challenge was initiated by administering $3 \times 10^{6}$ (Expt. 1 ) or $1 \times 10^{6}$ (Expts. $2 \& 3$ ) MOPC, MOPC/PSA/huCD25, or MOPC/PSMA cells s.c. Tumor size was measured by calipers and volume or area was calculated by multiplying length $\times$ width $\times$ height (Expts. 1\&3) or length $\times$ width (Expt. 2). At various time points post-vaccination (Fig 2), blood was obtained by tail vein bleeding and the plasma was evaluated for antihuman PSA antibody levels (see below).

For pre-existing tumor reduction experiments, male $\mathrm{BL} / 6$ mice were inoculated with $5 \times 10^{6}$ of TRAMP-C1/ PSA/IRES/huCD25 or TRAMP-C1/enGFP cells in Matrigel (BD) subcutaneously at the abdomen wall. Tumorinoculated animals were then immunized two times (at days 7 and 37) by intraperitoneal delivery of $4 \times 10^{5}$ PSA/IRES/huCD25-transduced or nontransduced DCs. Tumor size was measured from day 10 by calipers, and volume was calculated by multiplying length $x$ width $\times$ height.

\section{Indirect ELISA to detect plasma anti-human PSA antibody}

The 96-well plates were coated with $1 \mu \mathrm{g} / \mathrm{ml}$ of human PSA antigen (Fitzgerald Industries International, Concord, MA) in PBS containing $0.05 \% \mathrm{NaN}_{3}$ (PBSN) and incubated at room temperature overnight. After blocking for 1 hour at $37^{\circ} \mathrm{C}$ by the addition of $10 \%$ rabbit serum diluted in PBSN, either a $2 \times$ dilution of a $1 \mu \mathrm{g} / \mathrm{ml}$ stock of PSA mAb (10-P20; Fitzgerald) in Blocking Buffer $(0.05 \%$ Tween 20 and $0.25 \%$ BSA in PBSN), as a standard, or $50 \mu \mathrm{l}$ of $1000 \times$ diluted mouse plasma samples in Blocking Buffer, as tests, were added to the plates and incubated overnight at room temperature. Plasma from naive female and male BALB/c mice was used as controls. A single sample was tested from each animal at each time point. The plates were then blocked again by a 1 hour incubation at $37^{\circ} \mathrm{C}$ in $10 \%$ sheep serum, and then further incubated with goat anti-mouse IgG conjugated to alkaline phosphatase (Sigma) for 5 hours at room temperature. After incubation with substrate (pNPP), 
qualitative hydrolysis of NPP was detected using a microtiter plate reader (SOFTmax 2.32) with a 405-nm filter. Prebleeds of recipient animals provided baseline levels. Dilutions were also made using blocking buffer to re-assay samples that were beyond linearity for the initial $1000 \times$ dilution.

\section{Tumor-reactive $T$ cell cytokine secretion assay}

On day 5 after the second DC vaccination, spleens were harvested from some recipient mice and a single-cell suspension was generated. RBC were lysed in ACK buffer (Quality Biologics; Gaithersburg, MD) according to the manufacturer's protocol, and the resultant mononuclear cells plated at $0.5 \times 10^{6} / \mathrm{ml}$ in RPMI 1640 (Mediatech; Herndon, VA) with L-glutamine, $10 \%$ FCS, penicillin/ streptomycin, and 2-mercaptoethanol in six-well culture plates. Splenocytes were cocultured for 20 hours with either unmodified MOPC, MOPC/PSA/huCD25, MOPC/ PSMA, or $\mathrm{MOPC} / \alpha$-gal $\mathrm{A} / \mathrm{huCD} 25$ at a spleen cell to tumor cell ratio of $2: 1$. Evaluation of IFN- $\gamma$ secretion by splenocytes was performed according to manufacturer's instructions (Miltenyi). Briefly, $10^{6}$ cells were washed and labeled with each antibody (bispecific anti-CD $45 \times$ antiIFN- $\gamma$ ) on ice for 5 minutes. Cells were then incubated at $37^{\circ} \mathrm{C}$ for 45 minutes to allow cytokine secretion and capture. Cells were harvested, washed, and stained with either anti-IFN- $\gamma$ PE or an isotype-matched PE-labeled antibody (BD). At this time, cells in each tube were stained with both anti-CD4 FITC and anti-CD8 APCconjugated antibodies (BD). After washing (Miltenyi wash buffer, $2 \mathrm{mM}$ EDTA, $0.5 \%$ BSA), $2 \times 10^{3}-10^{4}$ live events per tube were acquired using a FACSCalibur ${ }^{\mathrm{TM}}$ instrument (BD). Dead cells were gated out using propidium iodide $(5 \mu \mathrm{g} / \mathrm{ml}$, Sigma). CD4 or CD8 cells that had increased IFN- $\gamma$ PE staining relative to the isotype control stain were determined to be positive for cytokine secretion. Data was analyzed using CELLQuest $^{\mathrm{TM}}$ software (BD).

\section{Results}

\section{Vector constructions and testing}

Two novel recombinant oncoretroviral gene transfer vectors were created. The first vector engineers overexpression of human PSA along with human CD25 (huCD25), which can act as a cell surface marker to facilitate isolation of positive cells and to follow infection of DCs. The second vector engineers transfer of the cDNA for human PSMA. Both vectors were packaged in ecotropic and amphotropic packaging cell lines and single-cell producer isolates were expanded and analyzed. Staining of producer cell lines with a specific antibody to PSMA or huCD25 followed by FACS analysis demonstrated overexpression of these surface antigens with an increase of mean fluorescence intensity of 1-2 logs (data not shown). Secreted human PSA levels were also measured from producer clones. The highest producing clones secreted $>1000 \mathrm{ng} / \mathrm{ml}$ PSA in the supernatant of 24 hour cultures (data not shown). Test infections of naïve NIH3T3 and HeLa cells with the ecotropic and amphotropic producer supernatants, respectively, were also performed. Vigorous surface expression of PSMA and huCD25 was observed on infected cells as was secretion of human PSA (data not shown). Overexpression of PSA/ huCD25 and PSMA in producer cells or following multiple infections of test cells also had no deleterious effects on cell growth (data not shown).

\section{Characterization of human PSA/huCD25- and PSMA- transduced primary murine DCS}

A pilot experiment to examine cell surface antigen staining patterns of murine BM-derived DCs under our infection and differentiation conditions was first undertaken. BMMCs were transduced separately five times with supernatant from E86/PSA/IRES/huCD25 \#21/1520 and E86/PSMA\#65. Mock-transduced cells were also maintained in analogous culture media. On day 6 after the initial BMMC harvest, cells were collected and counted. Starting with $\sim 10^{8}$ viable cells in each group after BMMC isolation, total viable cells in each group recovered 6 days later approximated $60 \%$ of input. Cells were then incubated for 1 day with $\operatorname{rmTNF}-\alpha$. To determine their characteristics, cells from days 0,6 , and 8 were costained for huCD25 or human PSMA cell surface expression along with a variety of murine cell surface markers. At day $6, \sim 75 \%$ of total cells in the PSA/huCD25-infected group were positive for huCD25 expression, whereas $50 \%$ of total cells in the PSMAinfected group were found to be positive for human PSMA expression (Table 1). From day 6 to day 8 a slight decrease in the total number of huCD25- and PSMApositive cells occurred (data not shown).

Our culture and transduction conditions upregulated a number of cell surface antigens typically expressed on functional DCs. Notably, mCD11b, mCD11c, mCD40,

Table 1 Percentages of live transduced murine BMMCs staining for transgene expression and various surface antigens

\begin{tabular}{|c|c|c|c|c|c|c|}
\hline \multirow[b]{3}{*}{ Markers } & \multirow[b]{3}{*}{ Day 0} & \multicolumn{5}{|c|}{ Day 6} \\
\hline & & \multirow[b]{2}{*}{ NT } & \multicolumn{2}{|c|}{ PSA/huCD25 } & \multicolumn{2}{|c|}{ huPSMA } \\
\hline & & & & $\begin{array}{l}\text { Double } \\
\text { positive }\end{array}$ & & $\begin{array}{l}\text { Double } \\
\text { positive }\end{array}$ \\
\hline huCD25 & $<1$ & $<1$ & 75 & & $<1$ & \\
\hline huPSMA & $<1$ & $<1$ & $<1$ & & 52 & \\
\hline mCD11b & 37 & 82 & 83 & 76 & 85 & 49 \\
\hline mCD11c & 4 & 49 & 47 & 45 & 45 & 38 \\
\hline mCD25 & $<1$ & 6 & 5 & 5 & 7 & 6 \\
\hline mCD40 & 0.6 & 3 & 3 & 3 & 7 & 7 \\
\hline mCD54 & 13.2 & 71 & 65 & 64 & 63 & 49 \\
\hline mCD80 (B7-1) & 11.5 & 37 & 23 & 22 & 27 & 24 \\
\hline mCD86 (B7-2) & 0.6 & 22 & 20 & 16 & 22 & 14 \\
\hline mDEC-205 & 7.5 & 20 & 18 & 17 & 9 & 8 \\
\hline mCD224 (NK cells) & $<1$ & $<1$ & $<1$ & $<1$ & 3 & 3 \\
\hline$m l-A^{d}$ & 10.2 & 33 & 30 & 27 & 36 & 29 \\
\hline
\end{tabular}


mCD54, mCD80, mCD86, mDEC-205, and $\mathrm{mI}^{\mathrm{d}} \mathrm{A}^{\mathrm{d}}$ expression levels were increased substantially at day 6 compared to day 0 input controls (Table 1). Importantly, a high percentage of cells expressing DC markers or costimulatory molecules also coexpressed huCD25 or PSMA (Table 1). Murine CD224 and mCD25 expression itself were not affected by the culture and transduction conditions and mMLy-6G expression was decreased. Little difference was observed in overall staining patterns between day 6 and day 8 cells (data not shown). A separate attempt to further mature transduced DCs in the presence of rmTNF- $\alpha$ in culture led to increased expression of key antigens at day 11 such as mCD54, mCD80, CD86, and mDEC-205 (data not shown).

\section{PSA/huCD25- and PSMA-transduced DC: in vitro MLR and $T$ cell sensitization assays}

Functional in vitro analyses of transduced and control murine DCs were first performed. First, we tested transduced DCs in an MLR assay. Various stimulatorto-effector cell ratios $(1: 1,1: 4,1: 16,1: 64,1: 256,1: 1024$, $1: 4096)$ were used in the presence and absence of unmanipulated, enriched allogeneic T cells. Murine DCs transduced with PSA/huCD25 and PSMA vectors potently sensitized allogeneic $\mathrm{T}$ cell proliferation as measured by levels of $\left[{ }^{3} \mathrm{H}\right]$ thymidine incorporation (Fig 1). In fact, stimulation of allogeneic $\mathrm{T}$ cells by all differentiated cells (including nontransduced controls) maintained under our culture and transduction conditions surpassed (by $>2 \operatorname{logs}$ in some cell ratios) the ability of allogeneic splenocytes to stimulate $\mathrm{T}$ cell proliferation. This result demonstrates that culture and transduction of these cells did not appear to decrease their allostimulatory function.

The PSA/huCD25- and PSMA-transduced DC were also capable of stimulating syngeneic $\mathrm{T}$ cells in vitro in a tumor antigen-specific manner. Syngeneic T cells that were initially stimulated with DC/PSA/huCD25 were capable of producing IFN $-\gamma$ in response to the PSA/ huCD25-transduced, but not the nontransduced MOPC

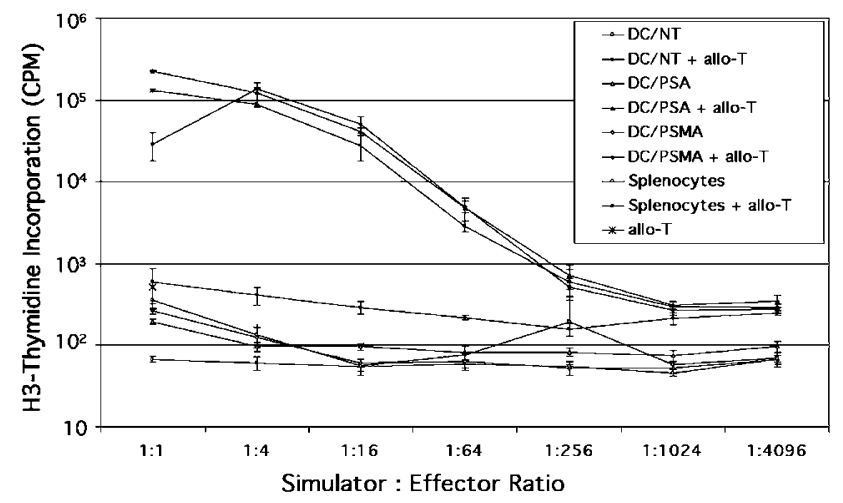

Figure 1 Allogeneic mixed lymphocyte reaction. The DCs and splenocytes, both of which were used as stimulators, were irradiated and plated in serial dilutions along with effector splenocytic T cells in 96-well tissue culture plates. DC/NT, nontransduced DC; allo-T, allogeneic splenocytic T cells. tumor line (data not shown). Interestingly, the magnitude of PSA/huCD25-specific cytokine release approached or exceeded that obtained with polyclonal restimulation employing anti-CD3/anti-CD28 beads. Similarly, syngeneic $\mathrm{T}$ cells that were initially stimulated with PSMAtransduced DC were capable of producing a high level of IFN- $\gamma$ ( $\sim 15$-fold over background) specifically in response to the PSMA-transduced MOPC tumor line. There was no evidence of specific $\mathrm{T}$ cell cytokine secretion of other cytokines tested, including IL-2, IL-4, IL-5, and IL-10 (data not shown).

We designed our PSA/huCD25 vector in a bicistronic format to facilitate isolation of optimal clonally derived packaging cell lines, to accurately quantitate vector titer, and for the possibility of enrichment of transduced cells prior to transplantation should transduction efficiencies be too low to engender specific immune responses. Fortunately, the latter was not the case (see below). Yet a question arises: Could the expression of the huCD25 marker be providing a selective growth advantage to transduced cells, especially in the presence of IL-2? To test this, we did a series of experiments looking at the proliferation index of control murine $\mathrm{T}$ cells, nontransduced DC-like murine cells, enGFP-transduced DC-like murine cells, and PSA/huCD25-transduced murine cells. We cultured and transduced cells as above. DC-like cells were then enriched for CD11c expression. Approximately $75 \%$ of cells after enrichment expressed CD11c and $>75 \%$ of those cells were positive for enGFP expression or huCD25 expression (data not shown). In parallel, control murine T cells were enriched for CD90 expression. Next, these cells were incubated in triplicate in three separate assays with varying levels of mIL-2 (from 0 to $\left.10^{5} \mathrm{IU} / \mathrm{ml}\right)$. As expected, murine $\mathrm{T}$ cells demonstrated increased proliferation as measured by MTT assay in response to added levels of muIL-2 (data not shown). In sharp contrast, all DC-like cell cultures including controls, enGFP-expressing, and PSA/huCD25-expressing actually demonstrated distinct IL-2 dose-dependent growth inhibition (data not shown). Thus, the in vivo results (following) are not likely due to CD25-based amplification of DC-like cells.

\section{DC/PSA/huCD25 potently induce antibody-based immunity in vivo}

Next, we tested the ability of transduced cells to induce immunity in recipient animals. BMMCs were infected with either the PSA/huCD25- or the PSMA-expressing vectors. In this experiment, $\sim 75 \%$ of DC/PSA/huCD25 cell cultures stained positive for huCD25 expression, while $\sim 79 \%$ of DC/PSMA cells stained positive for PSMA (data not shown). Four mice received nontransduced DCs, 20 mice received DC/PSA/huCD25, and 20 mice received DC/PSMA. No untoward effects were observed in animals receiving either control or productively infected cells. At 2 weeks after the first immunization individual animals were bled and plasma collected for indirect ELISAs. After 2 weeks, animals received another immunization with cells that were $\sim 65 \%$ positive for 
huCD25 expression and $\sim 40 \%$ positive for PSMA expression. Again, no untoward effects were observed in the secondarily immunized animals.

At 1 week following the second immunization, animals were challenged with PSA/huCD25-expressing MOPC tumor cells (Expt. 1). We chose this tumor cell line for these initial studies designed to elucidate mechanisms because they are well-characterized and rapidly lead to tumors in animals. Figure 2 shows the results of the assay for the presence of anti-huPSA antibodies as measured by indirect ELISA. Anti-huPSA antibody levels, as measured against a known control, increased slightly over background due to the first immunization course (from 1 to $>50 \mu \mathrm{g} / \mathrm{ml}$ ) but then increased dramatically after the second immunization (to $\sim 2000 \mu \mathrm{g} / \mathrm{ml}$ ). The administration of specifically engineered MOPC/PSA/huCD25 tumor cells to animals did not significantly affect antibody titer (Fig 2). That said, a slight increase in anti-huPSA antibody titer was observed in week 7 (to $\sim 90 \mu \mathrm{g} / \mathrm{ml}$ ) in DC/PSMA immunized mice challenged with MOPC/PSA/huCD25 cells (Fig 2). These results demonstrate that the PSA/huCD25-transduced DCs, but not the PSMA-transduced DCs, generated a host B cellmediated anti-PSA response. Since antibody production requires $\mathrm{CD}^{+}{ }^{+} \mathrm{T}$ cell help, this result also indirectly indicates that the PSA/huCD25-transduced DC initiated a $\mathrm{CD} 4^{+} \mathrm{T}$ helper cell response in vivo.

DC/PSA/huCD25 and DC/PSMA vaccination induces tumor-specific $C D 8^{+} T$ cell immunity in vivo

To evaluate whether vaccinated mice generated $\mathrm{CD} 8^{+} \mathrm{T}$ cell immunity, specific release of IFN- $\gamma$ by recipient $\mathrm{T}$ cells was evaluated by cytokine capture flow cytometry. As Figure 3 a shows, a similar low frequency of splenic $\mathrm{CD} 8^{+}$ $\mathrm{T}$ cells from recipients of nontransduced DC (DC/NT) were capable of secreting IFN- $\gamma$ in response to each of the

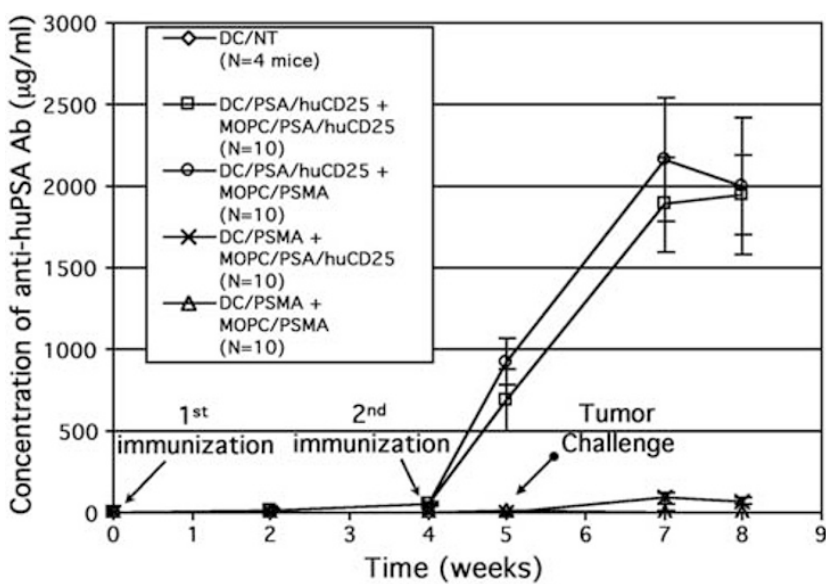

Figure 2 Titration of anti-human PSA antibody (IgG class) in plasma of DC/PSA/huCD25 and DC/PSMA immunized mice from Expt. 1 by indirect ELISA. Mice were immunized twice by i.p. delivery of $4 \times 10^{5}$ cells/mouse of DCs on weeks 0 and 4 . Tumor challenge was performed on week 5 . The mice were bled every 1 or 2 weeks after first immunization. DC/NT, nontransduced DC.
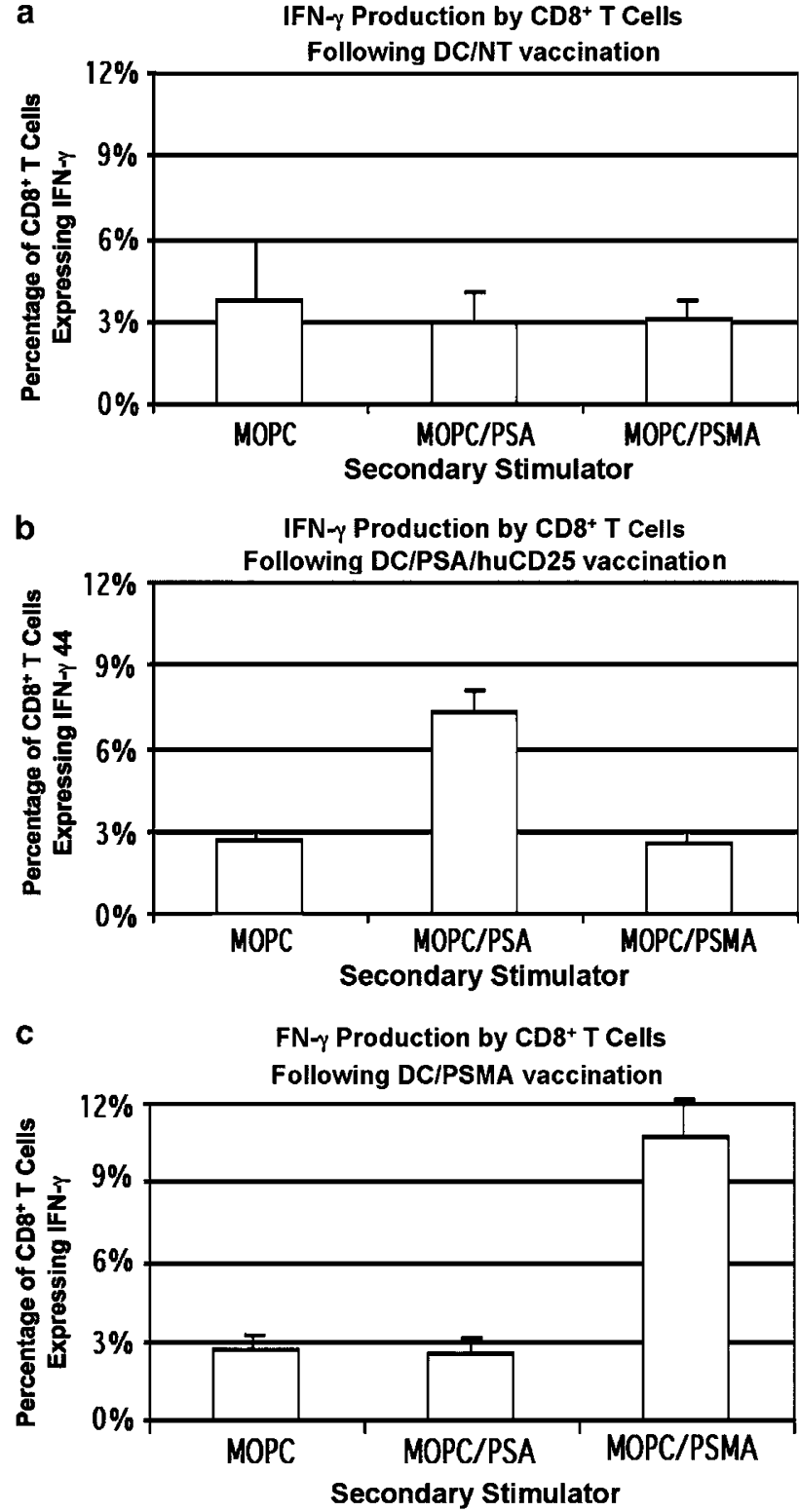

Figure 3 Gene-modified DC generate tumor-specific CD8 ${ }^{+} \mathrm{T}$ cells in vivo. $\mathrm{BALB} / \mathrm{c}$ mice received two vaccinations with either PSA/ huCD25-("DC/PSA/huCD25") or PSMA- ("DC/PSMA") transduced or nontransduced ("DC/NT") DC. On day 5 after the second vaccination, recipient splenocytes were stimulated for 20 hours in vitro with either PSA/huCD25- or PSMA-transduced or unmodified MOPC cells at a 2:1 spleen cell:tumor cell ratio. Cytokine capture flow cytometry was then performed to determine the frequency of IFN- $\gamma$ secreting cells by using a bispecific anti-CD $45 \times$ anti-IFN- $\gamma$ antibody in combination with anti-CD8 cell surface staining.

three tumor lines evaluated. In marked contrast, Figure $3 \mathrm{~b}$ shows that recipients of the $\mathrm{DC} / \mathrm{PSA} /$ huCD25 vaccination had a statistically significant increase in the frequency of $\mathrm{CD}^{+} \mathrm{T}$ cells capable of producing IFN- $\gamma$ in response to the relevant PSA/huCD25-transduced, but not PSMAtransduced tumor line $(P<.02)$. Similarly, as shown in Figure $3 c$, recipients of the DC/PSMA vaccination had a statistically significant increase in the frequency of $\mathrm{CD}^{+}$ 
$\mathrm{T}$ cells capable of IFN- $\gamma$ secretion in response to the relevant PSMA-transduced, but not PSA/huCD25-transduced, tumor line $(P<.02)$. The frequency of recipient CD4 ${ }^{+}$T cells capable of tumor-specific secretion of IFN$\gamma$ also was greater in both the DC/PSA/huCD25- and DC/ PSMA vaccination cohorts, although these differences did not reach statistical significance (data not shown).

\section{Both DC/PSA/huCD25 and DC/PSMA vaccination protect recipient mice from relevant tumor challenge}

Tumor sizes were measured in individual recipient animals (and averaged) commencing on day 2 after the tumor challenge (Fig 4). In Expt. 1, animals were challenged 1 week after the second vaccination. Engineered MOPC cells used in this experiment were found to be $\sim 80$ and $\sim 95 \%$ positive for expression of huCD25 and PSMA, respectively (data not shown). By day 2, tumors had appeared in all animals. At day 4, tumor sizes in all animals had increased. At this point, though, pronounced differences in the tumor course emerged. For specifically immunized animals, that is, animals that received DC/PSA/huCD25 cells and were challenged with MOPC/PSA/huCD25 tumor cells (Fig 4a) or animals that
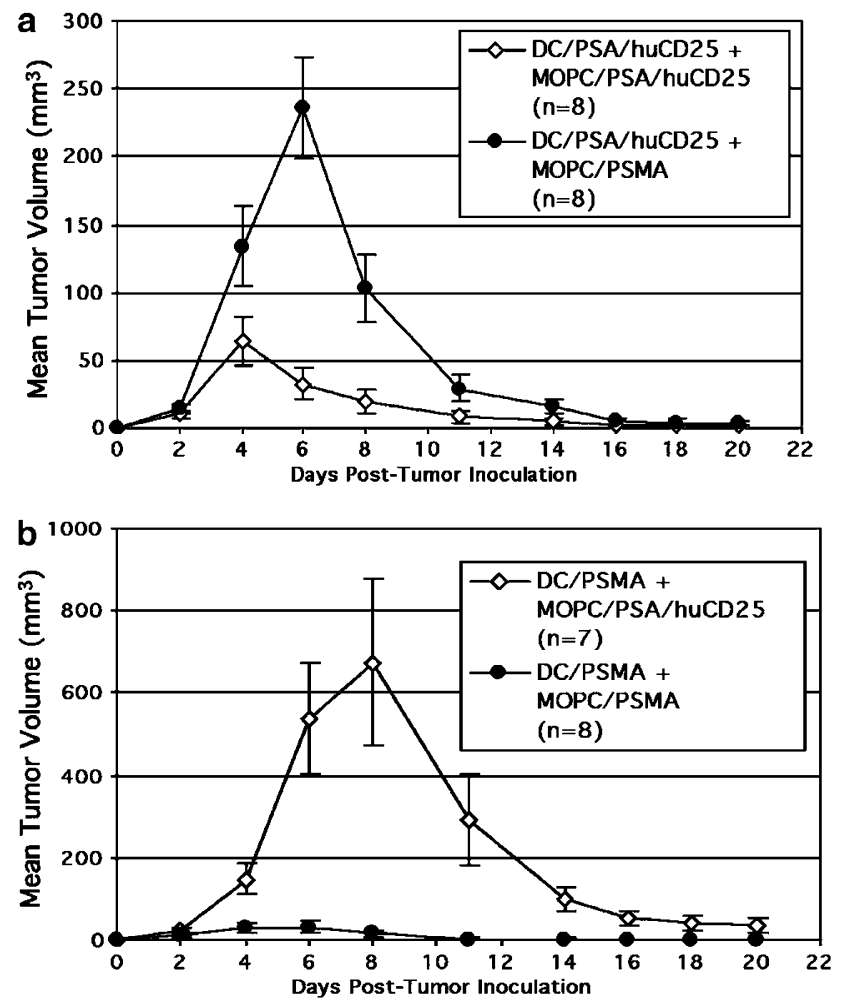

Figure 4 Inhibition of tumor growth in specific DC immunized mice. (a) PSA/huCD25 data from Expt. 1. Animals were immunized two times (separated by 4 weeks) by i.p. delivery of $4 \times 10^{5} \mathrm{DC} / \mathrm{PSA} /$ huCD25 cells and challenged 1 week later by s.c. delivery of $3 \times 10^{6}$ cells/mouse of PSA/huCD25- or PSMA-expressing MOPC cells. (b) PSMA data from Expt. 1. Animals were immunized two times (separated by 4 weeks) by i.p. delivery of $4 \times 10^{5}$ DC/PSMA cells and challenged as in (a). received DC/PSMA and were challenged with MOPC/ PSMA (Fig 4b), tumor sizes decreased sharply from this point forward. Indeed tumors became almost undetectable in most animals in these groups. In contrast, tumors in control animals continued to increase and reached large sizes before decreasing at later dates.

We next repeated these experiments with more controls and with a longer period (18 weeks) between the second immunization and the tumor challenge (Expt. 2). Similar results were obtained in that specific tumors were reduced or became nonexistent much faster than controls (data not shown). Additional controls, including animals receiving nontransduced but cultured DCs as the immunogens and nonimmunized normal animals receiving MOPC/PSA/huCD25 and MOPC/PSMA tumor cells, were also performed. We also immunized animals either s.c. or i.p. In this experiment, three-fold less tumor cells were administered to recipient animals compared to Expt. 1. Also, a lower percentage of these tumor cells were actually expressing huCD25 $(\sim 56 \%)$. Peak tumor size in this experiment occurred a little later, at days 13-17 and we did observe a slightly better response to tumor challenges when the transduced DCs were administered i.p. rather than s.c. (data not shown). We tracked antihuPSA antibody production in this experiment as well and observed a similar initial pattern as before. By 18 weeks, the antibody titer decreased three-fold although it remained substantially above background levels (data not shown). The anti-PSA antibody titer was restored after specific MOPC/PSA/huCD25 tumor challenge and actually surpassed earlier levels (data not shown).

Lastly, since Expts. 1 and 2 were performed with female mice only (both donors and recipients), we sought to examine immunization effects in male mice (Expt. 3). In humans, there are three glandular kallikrein genes (including PSA) while in mice there are 24 genes with 14 presumed to be functional. ${ }^{30} \mathrm{We}$ also sought to further explore our use of huCD25 as a cell surface marker in this model. Male and female mice were vaccinated with transduced male or female DCs, respectively, as in Expt. 1. In this Expt. (number 3), a lower percentage of DCs were positive for expression of huCD25 $(<20 \%$; data not shown). Nonetheless, with fewer numbers of effective specifically transduced DCs, the vaccination strategy was still quite effective and robust protection responses were observed. Figure 5a shows the results of the studies with vaccinated male mice. As can been seen, the DC/PSA/ huCD25-vaccinated male mice clear specific tumors nearly as effectively as their female counterparts and much more effectively than controls.

We also collected spleens from four randomly-selected female animals in each group shortly after the second vaccination. Figure $5 b$ shows the results of the cytokine capture flow cytometry assay examining the frequency of $\mathrm{CD}^{+} \mathrm{T}$ cells secreting IFN- $\gamma$ specifically in response to relevant antigen-expressing tumor cells. As Figure 3 shows, an increased frequency of cytokine-secreting $\mathrm{CD}^{+}$cells was observed in specifically vaccinated animals relative to controls $(P<.03)$. Importantly, $\mathrm{CD}^{+}$cell INF- $\gamma$ secretion in response to $\alpha$-gal $\mathrm{A} /$ 
548
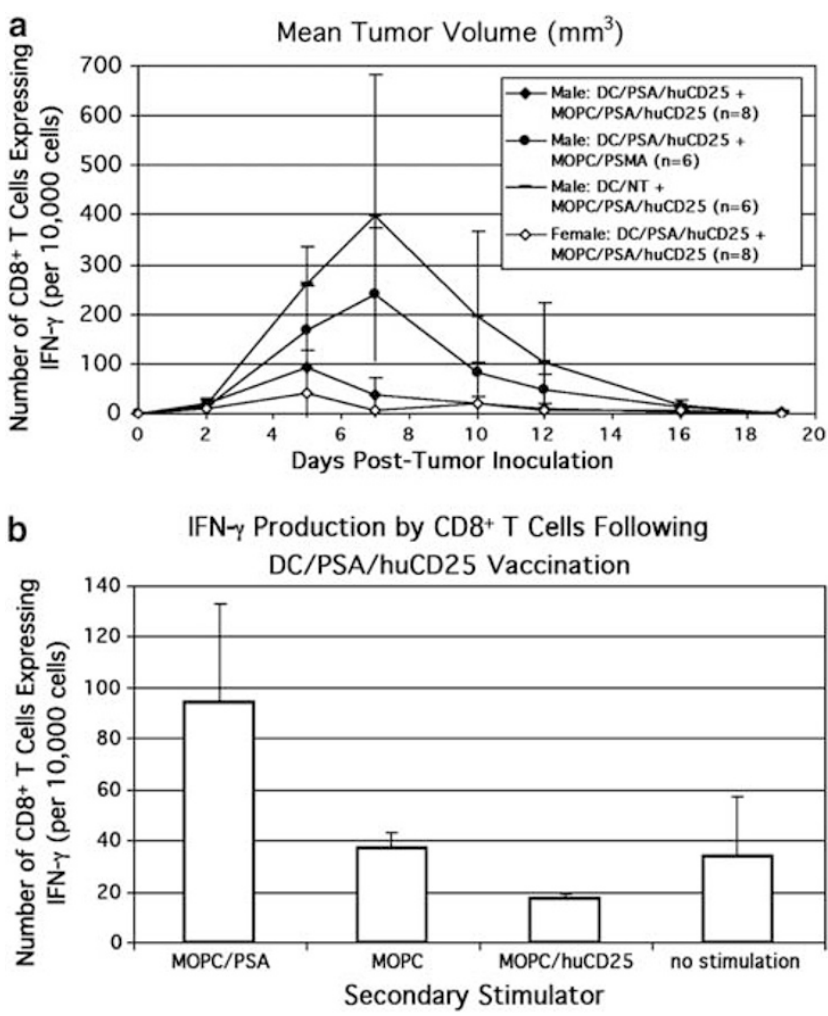

Figure 5 Tumor protection in male and $\mathrm{CD}^{+} \mathrm{T}$ cell responses in vaccinated female mice. (a) PSA/huCD25 data from Expt. 3. Male (and female control) animals were immunized two times (separated by 4 weeks) by i.p. delivery of $4 \times 10^{5} \mathrm{DC} / \mathrm{PSA} / \mathrm{huCD} 25$ or control cells and challenged 1 week later by s.c. delivery of $1 \times 10^{6}$ cells/ mouse of PSA/huCD25- or PSMA-expressing MOPC cells. (b) Lack of huCD25-specific $\mathrm{CD}^{+}{ }^{+} \mathrm{T}$ cells that secrete IFN- $\gamma$ in vivo in female mice. Balb/c mice received two vaccinations with DC/PSA/huCD25. On day 5 after the second vaccination, recipient splenocytes were stimulated for 20 hours in vitro with either MOPC/PSA/huCD25, MOPC/PSMA, MOPC/ $\alpha$-gal A/huCD25, or parent MOPC cells at a 2:1 spleen cell:tumor cell ratio. Cytokine capture flow cytometry was then performed to determine the frequency of IFN- $\gamma$-secreting cells by using a bispecific anti-CD $45 \times$ anti-IFN $-\gamma$ antibody in combination with anti-CD8 cell surface staining.

huCD25-transduced MOPC cells was not increased relative to nontransduced MOPC cells. This result strongly indicates that the augmented antitumor immunity in DC/PSA/huCD25 vaccinated mice is directed against the PSA component and not the cell surface marker.

\section{Reduction of specific murine prostate cancer epithelial cell tumors}

To gauge effects of our immuno-gene therapy approach using relevant murine prostate cancer cells and to determine whether we could actually reduce pre-existing tumors by implementation of this strategy, we performed another experiment. We injected animals with TRAMPC1/PSA-huCD25 or TRAMP-C1/enGFP (control) cells. After 7 days, we inoculated animals with specifically transduced or control DCs. Tumors were measured and

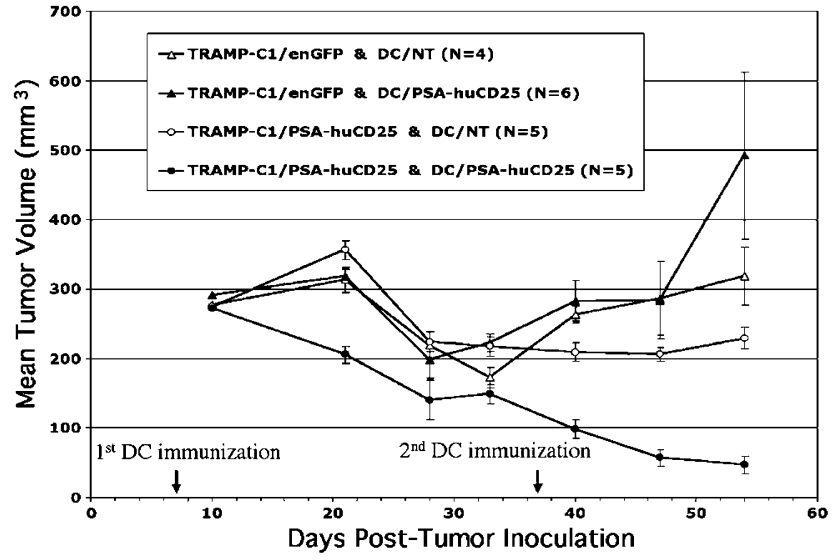

Figure 6 Specific growth inhibition of pre-existing tumors derived from TRAMP-C 1 cells by DC immunization in $\mathrm{BI} / 6$ mice. Tumor inoculation was performed at Day 0 by s.c. delivery of $5 \times 10^{6} \mathrm{PSA} /$ huCD25-expressing TRAMP-C1 cells or parent TRAMP-C1 cells. Tumor inoculated animals were then immunized two times at Day 7 and 37 by i.p. delivery of $4 \times 10^{5} \mathrm{PSA} /$ huCD25-transduced or nontransduced DCs.

volumes were calculated as above. After the first DC inoculation, we observed a substantial reduction in tumor sizes when specifically transduced DCs were administered to animals bearing specific-antigen-expressing tumors (Fig 6). This effect was even more pronounced leading to a further disparity in tumor sizes after a second inoculation on day 37 post-tumor inoculation. Indeed at day 52, PSA-huCD25 tumors were almost completely eradicated, while tumors in control animals were actually increasing in size (Fig 6).

\section{Discussion}

The successful immunotherapy of cancer, including prostate cancer, likely will require favorable presentation of tumor antigens to host T cells along MHC Class I- and Class II-restricted pathways that induce both antibody and cell-mediated immunity. DC-based immunization has been studied as an approach to sensitize host immunity towards tumor antigens, with several investigators previously evaluating transfer of whole genes into DC as a method for vaccination. In this report, we demonstrate that coding sequences for human prostate antigens PSA and PSMA can be successfully transferred via clinically relevant recombinant oncoretroviruses into hematopoietic cells that have the capacity for maturation in vitro into cells that express DC cell surface antigens and have APC function. These gene-modified cells potently induced both antibody and cell-mediated immunity in vivo in syngeneic hosts, with resultant tumor-specific protection against tumor cell challenge. Our results extend the findings of prior investigators evaluating gene transfer as a mode of immunization such as for a melanoma antigen ${ }^{31}$ into a model that has potential relevance to prostate cancer. Further, we also suggest here that this approach appears 
to promote both Class I- and Class II- restricted immunity in vivo.

In this study, we observed an induction of both antibody and cell-mediated immunity directed against prostate tumor antigens. Of particular note was the high level of human PSA-specific antibody titer we observed after only one vaccination with the transduced DCs that was even further elevated after a second vaccination. We also observed a significant frequency of $\mathrm{CD}^{+} \mathrm{T}$ cells capable of IFN- $\gamma$ production in response to specific prostate tumor antigen-expressing cancer cells. This appeared to be a particularly robust antitumor response, as the frequency of $\mathrm{CD}^{+}$cells capable of producing IFN- $\gamma$ in response to relevant tumor was similar to the IFN- $\gamma$ response to polyclonal CD3/CD28 antibody stimulation. Future studies will address effector mechanisms in detail by using immune molecule gene-deficient host animals, such as for IFN- $\gamma$ for example, to evaluate the contribution of this molecule to the observed protection from tumor challenge or by engineering in vivo $\mathrm{T}$ cell subset depletion with administration of specific antibodies.

In our study, we also observed that transgene-modified DC vaccination provided a long-lasting memory response, with specific tumor protection preserved for at least 18 weeks after final DC vaccination. Future studies in this area will utilize secondary $\mathrm{T}$ cell transfer to better elucidate the specific populations responsible for the observed immunologic memory. We also found that PSA/ huCD25 transgene-modified DC vaccination was effective in both female and male recipients. This observation suggests that even in male mice, which continually see orthologues of our transgene products, antigen presentation through a DC vaccine approach is effective. Lastly, we even observed reduction of pre-existing tumors in a model system with more relevance to prostate cancer.

The mechanism whereby MOPC tumor size decreased in all treated and control $\mathrm{BALB} / \mathrm{c}$ recipients is not known as we were unable to demonstrate antibody or $\mathrm{T}$ cell specificity to the tumor alone in such recipients using our current assays (data not shown). Nonetheless, our data are clear that tumor clearance is much faster in specifically immunized animals - if they even grow tumors at all. This endogenous antitumor response in our current model will need to be rectified for more detailed mechanistic studies. Future models here will involve the use of lethally-irradiated animals and adoptive transfer as we have recently shown that this endogenous protection can be fully abrogated by this approach (data not shown).

In this study, we obtained efficient functional gene transfer into capable murine APCs. Indeed, we added a cell surface marker (huCD25) in a bicistronic format to our PSA vector to allow possible preselection ${ }^{10}$ of transduced cells prior to immunization. However, because of the high frequency of DC transduction using our method, such an enrichment procedure was not necessary in our studies. Our bicistronic vector may be more relevant to the translation of our approach to the human system, as the efficiency of gene transfer into primitive primate hematopoietic cells is reduced. Nonetheless, this huCD25 marker will be of value in further murine studies, as we have demonstrated that this marker and available antibody sets have no background staining in murine recipients (Qin et $\mathrm{al}^{10}$ and Medin, unpublished data). As such, one may utilize this approach to track transduced and transplanted DCs in vivo to evaluate cell migration patterns, longevity, and optimal routes of administration. Importantly, using this bicistronic approach, we observed that the intracellular and secreted gene product (PSA) was presented to the immune system, whereas the surface marker gene (huCD25) did not generate a detectable immune response. This finding would appear to represent a technical advance with potential therapeutic advantages, as some other gene marking approaches such as fluorescence proteins can be immunogenic. Furthermore, our polycistronic vector approach also allows one the flexibility to construct dual- (or even triple ${ }^{32}$ ) function therapeutic vectors that coexpress other molecules such as cytokines that can further impact the immune response. The use of two prostate antigen-expressing vectors also allows combinatorial infections of the same APC, thereby increasing the repertoire of tumor antigens presented to the immune system.

The whole prostate antigen, DC-based strategy we have developed likely compares favorably to other vaccine approaches, such as "peptide loading". This approach, where peptides are added to autologous DCs in culture that complex with the MHC molecules, has been pursued by a number of groups. Clinical protocols have been undertaken using this strategy in melanoma, ${ }^{33}$ follicular B-cell lymphoma, ${ }^{34}$ and prostate cancer $^{35}$ with limited effectiveness. Several factors may contribute to the general lack of therapeutic impact of this approach. Peptide selection may not be optimal, either in binding affinity for the MHC molecule or in initiating a multifaceted $\mathrm{T}$ cell response. The half-life for the peptideMHC complex is also often reduced, possibly requiring multiple infusions of loaded DCs or boosting by injection of the antigen itself. ${ }^{34}$ Lastly, this approach is haplotype restricted and the use of only one peptide may actually lead to tolerance to the parent antigen. ${ }^{36}$ Our DC-based whole gene approach, which appeared to rapidly induce antibody and cell-mediated immunity along with immunologic memory, may overcome some of these limitations inherent to the peptide loading method.

Similarly, the whole prostate antigen oncoretroviral gene transfer approach we have developed may compare favorably to approaches using whole tumor cells as vaccines $^{37}$ or methods using alternative gene delivery vehicles. Indeed, a variety of gene transfer vectors have been used for this goal including recombinant adenovirus, lentiretrovirus, and vaccinia virus. While transfer into some hematopoietic cells using these delivery methods is quite efficient, ${ }^{38}$ long-term expression is often limited or repeat administration is not possible due to host immune neutralization of the delivery vehicle. Our use of oncoretroviral vectors may be advantageous relative to recombinant adenoviral approaches, for example, as this approach has been shown to actually suppress the 
capacity of T cells to be stimulated by transduced DC. ${ }^{39} \mathrm{It}$ is possible that lentiretroviral vectors may be amenable to our whole gene DC-based vaccination strategy, as these vectors can transfer genes into mature, nondividing DC. ${ }^{40}$ However, recombinant lentiretroviruses have only been approved for clinical use in one trial to date and largescale vector production issues remain, along with the fact that mature DCs themselves can be in limiting numbers or difficult to isolate from prostate cancer patients.

These initial murine studies indicate that our recombinant oncoretroviral whole gene vaccination approach may represent a promising future modality in the immune therapy of prostate cancer. Also, as shown above, we have packaged our PSA and PSMA recombinant vectors in amphotropic packaging cell lines to allow eventual infection of human bone marrow-derived CD34 + cells. In preliminary studies, we have demonstrated that an enGFP marking vector can infect these human hematopoietic stem/progenitor cells quite efficiently leading to transgene expression in differentiated cells that have a distinct DC morphology (data not shown). In vitro experiments will then be performed to determine whether DCs from normal volunteers and from prostate cancer patients can be infected and engineered to effectively present PSA and PSMA. These further murine and human studies will hopefully develop a rationale for pilot clinical studies using oncoretrovirally transduced DC in the near future for the immune therapy of prostate cancer.

\section{Acknowledgments}

We thank Christopher Siatskas (OCI, UHN) for helpful discussions and critical reading of the manuscript along with JingMei Liu (UIC), Gangjian Qin (UIC), and John Campbell (OCI, UHN) for experimental assistance. This study was supported in part by the US Department of Defense Grant \#DAMD17-00-1-0083 (to JAM).

\section{References}

1. Vesalainen S, Lipponen P, Talja M, Syrjanen K. Histological grade, perineural infiltration, tumour-infiltrating lymphocytes and apoptosis as determinants of long-term prognosis in prostatic adenocarcinoma. Eur $J$ Cancer. 1994;30A:1797-1803.

2. Wei C, Storozynsky E, McAdam AJ, et al. Expression of human prostate-specific antigen (PSA) in a mouse tumor cell line reduces tumorigenicity and elicits PSA-specific cytotoxic T lymphocytes. Cancer Immunol Immunother. 1996;42:362368.

3. Liu KJ, Chatta GS, Twardzik DR, et al. Identification of rat prostatic steroid-binding protein as a target antigen of experimental auto-immune prostatitis: implications for prostate cancer therapy. J Immunol. 1997;159:472-480.

4. Banchereau J, Steinman RM. Dendritic cells and the control of immunity. Nature. 1998;392:245-252.

5. Steinman RM. The dendritic cell system and its role in immunogenicity. Ann Rev Immunol. 1991;9:271-296.
6. Aalamian M, Pirtskhalaishvili G, Nunez A, et al. Human prostate cancer regulates generation and maturation of monocyte-derived dendritic cells. Prostate. 2001;46: 68-75.

7. Pirtskhalaishvili G, Shurin GV, Gambotto A, et al. Transduction of dendritic cells with $B c 1-x_{L}$ increases their resistance to prostate cancer-induced apoptosis and antitumor effect in mice. $J$ Immunol. 2000;165: 1956-1964.

8. Troy A, Davidson P, Atkinson C, Hart D. Phenotypic characterisation of the dendritic cell infiltrate in prostate cancer. J Urol. 1998;160:214-219.

9. Takenaka T, Murray GJ, Qin G, et al. Long-term enzyme correction and lipid reduction in multiple organs of primary and secondary transplanted Fabry mice receiving transduced bone marrow cells. Proc Natl Acad Sci USA. 2000;97:7515-7520.

10. Qin G, Takenaka T, Telsh $\mathrm{K}$, et al. Pre-selective gene therapy for Fabry disease. Proc Natl Acad Sci USA. 2001;98:3428-3433.

11. Henderson RA, Nimgoankar MT, Watkins SC, et al. Human dendritic cells genetically engineered to express high levels of the human epithelial tumor antigen mucin (MUC1). Cancer Res. 1996;56:3763-3770.

12. Reeves ME, Royal RE, Lam JS, et al. Retroviral transduction of human dendritic cells with a tumor-associated antigen gene. Cancer Res. 1996;56:5672-5677.

13. Song ES, Lee V, Surh CD, et al. Antigen presentation in retroviral vector-mediated gene transfer in vivo. Proc Natl Acad Sci USA. 1997;94:1943-1948.

14. Specht JM, Wang G, Do MT, et al. Dendritic cells retrovirally transduced with a model antigen gene are therapeutically effective against established pulmonary metastases. J Exp Med. 1997;186:1213-1221.

15. Parajuli P, Mosley RL, Pisarev V, et al. Flt3 ligand and granulocyte-macrophage colony-stimulating factor preferentially expand and stimulate different dendritic and T cell subsets. Exp Hematol. 2001;29:1185-1193.

16. Ward AM, Catto JWF, Hamdy FC. Prostate specific antigen: biology, biochemistry and available commercial assays. Ann Clin Biochem. 2001;38:633-651.

17. Xue BH, Zhang Y, Sosman JA, Peace DJ. Induction of human cytotoxic T lymphocytes specific for prostate-specific antigen. Prostate. 1997;30:73-78.

18. Kim JJ, Trivedi NN, Wilson DM, et al. Molecular and immunological analysis of genetic prostate specific antigen (PSA) vaccine. Oncogene. 1998;17:3125-3135.

19. Heiser A, Maurice MA, Yancey DR, et al. Induction of polyclonal prostate cancer-specific CTL using dendritic cells transfected with amplified tumor RNA. J Immunol. 2001;166:2953-2960.

20. Gregorakis AK, Holmes EH, Murphy GP. Prostate-specific membrane antigen: current and future utility. Sem Urol Oncol. 1998;16:2-12.

21. Tasch J, Gong M, Sadelain M, Heston WDW. A unique folate hydrolase, prostate-specific membrane antigen (PSMA): a target for immunotherapy? Crit Rev Immunol. 2001;21:249-261.

22. Kim J, Palmer, JL, Finn L, et al. The pattern of serum markers in androgen-independent adenocarcinoma of the prostate. Urologic Oncol. 2000;5:97-103.

23. Chang SS, Reuter VE, Heston WD, et al. Five different antiprostate-specific membrane antigen (PSMA) antibodies confirm PSMA expression in tumor-associated neovasculature. Cancer Res. 1999;59:3192-3198. 
24. Mincheff M, Tchakarov S, Zoubak S, et al. Naked DNA and adenoviral immunizations for immunotherapy of prostate cancer: a phase I/II clinical trial. Eur Urol. 2000;38:208-217.

25. Henttu P, Vihko P. cDNA coding for the entire human prostate specific antigen shows high homologies to the human tissue kallikrein genes. Biochem Biophys Res Commun. 1989;160:903-910.

26. Takenaka T, Qin G, Brady RO, Medin JA. Circulating $\alpha$ galactosidase A derived from transduced bone marrow cells: relevance to gene therapy for Fabry disease. Hum Gene Ther. 1999;10:1931-1939.

27. Gehring U, Mohit B, Tomkins GM. Glucocorticoid action on hybrid clones derived from cultured myeloma and lymphoma cell lines. Proc Natl Acad Sci USA. 1972;69: 3124-3127.

28. Foster BA, Gingrich JR, Kwon ED, et al. Characterization of prostatic epithelial cell lines derived from transgenic adenocarconoma of the mouse prostate (TRAMP) model. Cancer Res. 1997;57:3325-3330.

29. Garlie NK, Lefever AV, Siebenlist RE, et al. T cells co-activated with immobilized anti-CD3 and anti-CD28 as potential immunotherapy for cancer. $J$ Immunother. 1999;22:336-345.

30. Olsson AY, Lundwall A. Organization and evolution of the glandular kallikrein locus in Mus musculus. Biochem Biophys Res Comm. 2002;299:305-311.

31. Lapointe R, Royal RE, Reeves ME, et al. Retrovirally transduced human dendritic cells can generate $\mathrm{T}$ cells recognizing multiple MHC Class I and Class II epitopes from the melanoma antigen glycoprotein 100. J Immunol. 2001;167:4758-4764.
32. Fan L, Owen JS, and Dickson, G. Construction and characterization of polycistronic retrovirus vectors for sustained and high-level co-expression of apolipoprotein A-I and lecithin-cholesterol acyltransferase. Atherosclerosis. 1999;147:139-145.

33. Nestle FO, Alijagic S, Gilliet $M$, et al. Vaccination of melanoma patients with peptide-or tumor lysate-pulsed dendritic cells. Nat Med. 1998;4:328-332.

34. Hsu FJ, Benike C, Fagnoni F, et al. Vaccination of patients with B-cell lymphoma using autologous antigen-pulsed dendritic cells. Nat Med. 1996;2:52-58.

35. Tjoa BA, Simmons SJ, Elgamal A, et al. Follow-up evaluation of a phase II prostate cancer vaccine trial. Prostate. 1999;40:125-129.

36. Huang YM, Yang JS, Xu LY, et al. Autoantigen-pulsed dendritic cells induce tolerance to experimental allergic encephalomyelitis (EAE) in Lewis rats. Clin Exp Immunol. 2000;122:437-444.

37. Nouri-Shirazi M, Banchereau J, Bell D, et al. Dendritic cells capture killed tumor cells and present their antigens to elicit tumor-specific immune responses. J Immunol. 2000;165: 3797-3803.

38. Medin JA, Karlsson S. Viral vectors for gene therapy of hematopoietic cells. Immunotech. 1997;3:3-19.

39. Jonuleit H, Tuting T, Steitz J, et al. Efficient transduction of mature $\mathrm{CD} 83^{+}$dendritic cells using recombinant adenovirus suppressed $\mathrm{T}$ cell stimulatory capacity. Gene Ther. 2000; 7:249-254.

40. Dyall J, Latouche JB, Schnell S, Sadelain M. Lentivirustransduced human monocyte-derived dendritic cells efficiently stimulate antigen-specific cytotoxic $\mathrm{T}$ lymphocytes. Blood. 2001;97:114-121. 